\title{
Coupling of semiconductors for photocatalytic oxidation and CO2 reduction reactions under visible-light
}

\author{
Hark Jin Kim and Wan In Lee* \\ Department of Chemistry, Inha University, Incheon, 402-752, Republic of Korea \\ (wanin@inha.ac.kr)
}

\begin{abstract}
Removal of environmental pollutants through photocatalytic reaction has drawn increasing attention over the last few decades. Photocatalysts have also been designed and investigated for the purpose of water splitting and $\mathrm{CO}_{2}$ reduction to generate clean energies. We fabricated several heterojunction structures between $\mathrm{TiO}_{2}$ and other visible-light absorbing semiconductors, and found that relative energy band locations between $\mathrm{TiO}_{2}$ and sensitizer are a crucial factor in determining the efficiency of the photocatalytic reactions. First, we investigated several coupled structures of $\mathrm{TiO}_{2}$ and sensitizers, whose $\mathrm{VB}$ are lower than that of $\mathrm{TiO}_{2}$ (denoted to "type-B heterojunction"). With visible light irradiation, the electrons in the VB of the semiconductor are excited to its CB. Thereby, its VB is rendered partially vacant, and the electrons in the $\mathrm{VB}$ of $\mathrm{TiO}_{2}$ can be transferred to that of the semiconductor, since its $\mathrm{VB}$ is located at lower level. As a result, the holes generated in the $\mathrm{VB}$ of $\mathrm{TiO}_{2}$ have sufficient lifetime to initiate the photocatalytic oxidation reactions. Some of the coupled systems exhibited significantly higher photocatalytic efficiency than the typical $\mathrm{N}^{-} \mathrm{doped}^{\mathrm{TiO}} \mathrm{O}_{2}$ in decomposing gaseous 2-propanol and several organic pollutants in aqueous solution. For further enhancement of visible-light catalytic efficiency, we doubly combined the two different sensitizers with $\mathrm{TiO}_{2}$. That is, sensitizer-B with lower $\mathrm{VB}$ position than that of $\mathrm{TiO}_{2}$ was designed to be located in the core of the $\mathrm{TiO}_{2}$ structure, whereas sensitizer-A with higher $\mathrm{CB}$ was loaded onto the $\mathrm{TiO}_{2}$ surface. Under visible-light irradiation, both the electrons and holes are generated in CB and $\mathrm{VB}$ of $\mathrm{TiO}_{2}$, and these active species induces remarkably high photocatalytic efficiency in evolving $\mathrm{CO}_{2}$. Second, we also found that some of the coupled photocatalytic systems can be used for the reduction of $\mathrm{CO}_{2}$ under visible-light irradiation. We monitored the evolution of methanol and carbon monoxide, and it was also found that relative energy band positions between two semiconductors were critical for the photocatalytic $\mathrm{CO}_{2}$ reduction reactions.
\end{abstract}

